\title{
BMJ Open Catch-up-ESUS - follow-up in embolic stroke of undetermined source (ESUS) in a prospective, open-label, observational study: study protocol and initial baseline data
}

\author{
Katharina Feil (D) , ${ }^{1,2}$ Johanna Heinrich, ${ }^{1}$ Clemens Küpper, ${ }^{1}$ Katharina Müller, ${ }^{1}$ \\ Christoph Laub, ${ }^{1}$ Aenne S von Falkenhausen, ${ }^{3,4}$ Regina Becker, ${ }^{1}$ \\ Frank A Wollenweber, ${ }^{1,5}$ Stefan Kääb, ${ }^{3,4}$ Moritz F Sinner, ${ }^{3,4}$ Lars Kellert $^{1}$
}

To cite: Feil K, Heinrich J, Küpper C, et al. Catch-upESUS - follow-up in embolic stroke of undetermined source (ESUS) in a prospective, openlabel, observational study: study protocol and initial baseline data. BMJ Open 2019;9:e031716. doi:10.1136/ bmjopen-2019-031716

- Prepublication history for this paper is available online. To view these files, please visit the journal online (http://dx.doi. org/10.1136bmjopen-2019031716).

KF and JH contributed equally.

Received 15 May 2019 Revised 26 September 2019 Accepted 10 October 2019

D) Check for updates

(C) Author(s) (or their employer(s)) 2019. Re-use permitted under CC BY-NC. No commercial re-use. See rights and permissions. Published by BMJ.

For numbered affiliations see end of article.

Correspondence to Dr Lars Kellert; Lars.Kellert@med.unimuenchen.de

\section{ABSTRACT}

Introduction So far there is no uniform, commonly accepted diagnostic and therapeutic algorithm for patients with embolic stroke of undetermined source (ESUS). Recent clinical trials on secondary stroke prevention in ESUS did not support the use of oral anticoagulation. As ESUS comprises heterogeneous subgroups including a wide age-range, concomitant patent foramen ovale (PF0), and variable probability for atrial fibrillation (AF), an individualised approach is urgently needed. This prospective registry study aims to provide initial data towards an individual, structured diagnostic and therapeutic approach in ESUS patients.

Methods and analysis The open-label, investigatorinitiated, prospective, single-centre, observational registry study (Catch-up-ESUS) started in 01/2018. Consecutive ESUS patients $\geq 18$ years who give informed consent are included and will be followed up for 3 years. Stratified by age $<60$ or $\geq 60$ years, the patients are processed following a standardised diagnostic and treatment algorithm with an interdisciplinary design involving neurologists and cardiologists. Depending on the strata, patients receive a transesophageal echocardiogram; all patients receive an implantable cardiac monitor. Patients $<60$ years with PFO and without evidence of concomitant AF are planned for PFO closure within 6 months after stroke. The current diagnostic and therapeutic workup of ESUS patients requires improvement by both standardisation and a more individualised approach. Catch-up-ESUS will provide important data with respect to AF detection and PFO closure and will estimate stratified stroke recurrence rates after ESUS.

Ethics and dissemination The study has been approved by the responsible ethics committee at the Ludwig Maximilian University, Munich, Germany (project number 17-685). Catch-Up-ESUS is conducted in accordance with the Declaration of Helsinki. All patients will have to give written informed consent or, if unable to give consent themselves, their legal guardian will have to provide written informed consent for their participation. The first observation period of the registry study is 1 year, followed by the first publication of the results including follow-up
Strengths and limitations of this study

- Catch-up-ESUS is an observational registry study to assess the clinical data of embolic stroke of undetermined source (ESUS) patients and describes the implementation of a new, interdisciplinary (neurological-cardiological) treatment algorithm.

- The clinical stratification within the treatment algorithm is based on individual patient characteristics.

- The follow-up in ESUS patients will allow to determine the most promising mode of secondary stroke prevention.

- The data collection as part of clinical routine leads to the possibility of loss of data in the course of the study.

- Catch-up-ESUS is further limited due to the singlecentre data collection.

of the patients. Further publications will be considered according the predefined individual follow-up dates of the stroke patients up to 36 months.

Trial registration number Clinicaltrialsregister.gov registry (NCT03820375).

\section{INTRODUCTION}

For the last 25 years, ischaemic strokes have been classified according to the TOAST (Trial of Org 10172 in Acute Stroke Treatment) criteria, which were developed in the context of a randomised treatment trial including the following five stroke etiologies: large-artery atherosclerosis, small vessel occlusion, cardioembolism, stroke of other determined aetiology and stroke of undetermined aetiology also called 'cryptogenic' stroke.' However, cryptogenic strokes represented a heterogeneous subgroup of ischaemic strokes including strokes still unexplained after standard diagnostic workup and strokes without 
sufficient diagnostic clarification. Due to this unsatisfying situation, a more distinct definition of a subgroup of cryptogenic strokes was established in 2014 and was termed 'embolic stroke of undetermined source' (ESUS). ${ }^{2}$ ESUS criteria are defined as follows: non-lacunar brain infarction; no proximal cervical arterial stenosis; no cardioembolic source; no other aetiology (eg, vasculitis, cervical artery dissection). ${ }^{2}$ Although ESUS accounts for $20 \%-30 \%$ of all strokes, clear guidelines for diagnostic procedures and treatment regimens in those patients are still lacking. ${ }^{23}$

\section{Study rationale}

In the context of two recent randomised controlled trials, NAVIGATE-ESUS ${ }^{3}$ and RESPECT-ESUS, ${ }^{4}$ the topic of secondary prevention therapy of ESUS patients has been discussed. Both trials compared the efficacy and safety of oral anticoagulation (OAC) with rivaroxaban ${ }^{3}$ and dabigatran, ${ }^{4}$ respectively, versus acetylsalicylic acid (ASA) for secondary stroke prevention in ESUS patients and did not find differences in stroke recurrence rates per year (4.7\% rivaroxaban vs $4.7 \%$ ASA and $4.1 \%$ dabigatran vs $4.8 \%$ ASA).${ }^{34}$ However, patients treated with rivaroxaban presented with significantly higher annual bleeding rates compared with ASA-treated patients $(1.8 \%$ vs $0.7 \%)$ and the trial was stopped prematurely. ${ }^{3}$ Whereas a consensus on secondary stroke prevention in ESUS patients has yet to be reached, OAC is not recommended. Another unresolved question is the role of a patent foramen ovale $(\mathrm{PFO})$ in ESUS patients. A common finding in about $25 \%$ of the population, ${ }^{5}$ the prevalence of a PFO is even higher in ESUS patients and is found in up to $78 \%$ of patients depending on patient age. ${ }^{2} 67$ Several studies on PFO closure in patients with cryptogenic stroke at age $<60$ years compared with standard medical therapy recently showed an advantage of $\mathrm{PFO}$ closure with regard to stroke recurrence ${ }^{8-10}$ Last but not least, a substantial subgroup of ESUS might be due to underlying atrial fibrillation (AF) not detected by the standard diagnostic evaluation including 24 hours of ECG monitoring. ${ }^{11}$ So far, four randomised clinical trials have demonstrated that prolonged rhythm monitoring after cryptogenic stroke leads to a higher detection rate of AF. ${ }^{11-14}$ Depending on the modality and length of the prolonged cardiac monitoring, the $\mathrm{AF}$ detection rate was up to 10-fold higher compared with standard diagnostic regimens. ${ }^{11-14}$ When patients were selected based on an adjudicated higher risk of $\mathrm{AF}$, the $\mathrm{AF}$ detection rate of an implantable cardiac monitor (ICM) reached up to $40 \%$ within 1year after stroke. ${ }^{15}$ Based on these results, an extended search for AF in ESUS patients and a detailed characterisation and stratification for AF risk seems warranted. Yet, sustainable recommendations on both the modality and length of search for $\mathrm{AF}$, and on the selection of patients for screening are missing.

\section{Aims of the registry study}

Due to this lack of guideline recommendations in ESUS patients, we have established an interdisciplinary, neurological-cardiological algorithm with detailed recommendations on diagnostic and therapeutic procedures, stratified based on individual patient characteristics. Enrolled patients are followed up in our observational study registry for 3 years. The aims of our study are (1) to establish and evaluate an interdisciplinary, standardised diagnostic and therapeutic algorithm for ESUS patients; (2) to identify the most promising mode of secondary stroke prevention in ESUS patients, stratified according to the individual's risk profile; (3) to determine the $\mathrm{AF}$ rates among risk strata of ESUS patients by ICM; and (4) to systematically detect PFO in suitable ESUS patients, followed by monitoring for outcomes after PFO closure.

\section{METHODS AND ANALYSIS}

The study has been registered at www.clinicaltrialsregister. gov. Any important modifications in the protocol will be entered there.

\section{Study design}

The Catch-Up-ESUS registry is an open-label, investigatorinitiated, prospective, single-centre, observational registry study that started on 1 January 2018.

\section{Inclusion criteria}

The clinical data of consecutive ESUS patients $\geq 18$ years of age, who were treated in our hospital and gave their written informed consent, are collected. The patients are included during their hospital stay for the index stroke; if informed consent is missing during hospital stay (due to organisational reasons), patients are included during follow-up. The individual observation period for each patient is at least 36 months from the time of stroke. ESUS patients who were not treated in our centre for the index stroke are not included.

\section{Exclusion criteria}

Patients $\geq 18$ years of age who are not willing to give informed consent and patients with life expectancy $<3$ months.

\section{Patient and public involvement}

The development of the study rationale was based on the described studies on the treatment of ESUS patients, a relevant group of patients in the stroke care. Patients were not included in the design of the study or in the recruitment for the study. The individual treatment according the treatment algorithm is discussed with every single patient during the hospital stay as well as the follow-up. The dissemination of the results to study participants takes places by publication of the results of the registry study.

\section{Diagnostic workup and assessment in stroke patients}

The data collection occurs during the index hospital stay. A standard diagnostic workup is carried out in all stroke patients to ascertain stroke aetiology. 
Clinical and neurological assessment

Information about demographics, living situation, premorbid functional status, lifestyle habits, personal health and family history, and medication use prior to the stroke is obtained from the patient or next of kin. The cardiovascular risk factors are defined based on current guidelines. ${ }^{16}$ Clinical examination consists of a standardised neurological examination. Stroke severity is assessed by a certified stroke neurologist using the National Institutes of Health Stroke Scale (NIHSS). The modified Rankin Scale (mRS) and the Barthel Index serve as measures of functional status.

\section{Brain imaging}

Infarct localisation is confirmed by brain CT and/or brain MRI. Infarcts on MRI are defined as acute diffusion restrictions (hyperintensities) on diffusion-weighted images with corresponding hypointense areas on apparent diffusion coefficient maps. In addition, T2-weighted and fluidattenuated inversion recovery sequences are acquired. Where required, vascular imaging by CT-angiography (CT-A) or MRI-angiography is performed to exclude large-artery atherosclerosis and small vessel disease.

\section{Ultrasound}

Ultrasound examination includes assessment of extracranial and intracranial vessels by Doppler sonography and colour duplex sonography. Specifically, the following measures are obtained: plaque thickness, plaque localisation $(<1 \mathrm{~cm}$ proximal or distal to carotid bifurcation), plaque configuration (eccentric or concentric), echogenicity (hypoechoic, isoechoic or hyperechoic) and stenosis grade according to the North American Symptomatic Carotid Endarterectomy Trial criteria.

\section{Standard cardiac examination}

Cardiac examination required in all patients includes a standard 12-lead ECG, a 3-day Holter-ECG or automated atrial fibrillation detection on ECG monitoring for at least 72 hours, and a transthoracic echocardiogram.

\section{Blood tests}

Serum and plasma samples, including markers of inflammation (eg, C-reactive protein (CRP), Interleukin 6 (IL-6)), of glycemic status (eg, glycated hemoglobin (HbAlc)), of lipid status (eg, low-density lipoprotein (LDL), high-density lipoprotein (HDL)) and of cardiac congestion (eg, N-terminal prohormone of brain natruiretic peptide (NT-proBNP)), are obtained for laboratory investigations.

\section{Assessment of AF-related risk markers}

In addition, we assess the risk of $\mathrm{AF}$ in all patients. We consider the following findings as contributing to a higher risk of AF: a $\mathrm{CHA}_{2} \mathrm{DS}_{2}$-VASc score $>4$; a NT-proBNP $\geq 125 \mathrm{pg} / \mathrm{mL}$; an increased risk for paroxysmal $\mathrm{AF}$ suggested by automatic AF detection on rhythm monitoring; atrial ectopic runs detected by Holter ECG recordings; a transthoracic echocardiography finding of left atrial enlargement $\geq 45 \mathrm{~mm}$; a transesophageal echocardiography (TEE) finding of either spontaneous contrast in the left atrial appendage or a left atrial appendage flow $\leq 0.2 \mathrm{~m} / \mathrm{s}$. The CHA2DS2-VASc score is a clinical prediction score for estimating the risk of stroke in patients with non-rheumatic AF used to determined whether or not anticoagulation treatment is necessary. The CHADS2 score is determined by adding the points that correspond to the conditions present in the individual patient $(\mathrm{C}$ : congestive heart failure - 1 point, $\mathrm{H}$ : hypertension - 1 points, A: Age $\geq 75$ years - 1 point, D: diabetes mellitus - 1 point, S2: prior stroke, TIA or thrombembolism - 2 points). The CHA2DS2-VASc score is a refinement of CHADS2 score and extens the latter by including additional common stroke risk factors (age 65-74, female gender and vascular disease, futhermore age $\geq 75$ has extra weight with 2 points). The maximum CHA2DS2VASc score is $9 .{ }^{17}$ The threshold for NT-proBNP was chosen based onthe management guidelines for heart failure by the European Society ofCardiology. ${ }^{18}{ }^{19}$ Anincreased risk for $\mathrm{AF}$ is considered relevant in the presence of at least one ofthe listed factors. The Risk of Paradoxical Embolism- (RoPe) Score is also collected from all patients with a PFOin order to determine the probability that the stroke is related to the $\mathrm{PFO} .^{20}$

\section{Advanced diagnostic workup according to our interdisciplinary ESUS evaluation algorithm \\ Stratified evaluation by age group}

ESUS patients are stratified by age $<60$ years (group 1 ) or $\geq 60$ years (group 2 ) following recently published studies on PFO closure.

Evaluation of a relevant PFO by transcranial ultrasound and further cardiac assessment

Patients allocated to group 1 are screened for the presence of a PFO by both the highly sensitive echocardiography contrast agent-guided transcranial ultrasound ('bubble test') and by the highly specific echocaerdiography contrast agent-guided TEE. ${ }^{7}$ A relevant right-to-left shunt according to transcranial ultrasound is defined as $>10$ gas bubbles detected by Doppler-ultrasound in the middle cerebral artery. On TEE, a relevant PFO is diagnosed by either a strong visualised right-to-left shunt of echocaerdiographic contrast agent or a moderate visualised right-to-left shunt of echocardiographic contrast agent combined with the presence of an atrial septal aneurysm with $>10 \mathrm{~mm}$ unidirectional deviation or $\geq 15 \mathrm{~mm}$ bidirectional deviation.

\section{Additional diagnostic procedures and blood tests}

In addition to standard laboratory blood tests, the following additional blood tests are performed in selected patients depending on clinical decisions: cerebrospinal fluid collection; assessment of vasculitisrelated markers including C3, C4, ANA-titer and differentiation, rheumatoid factor, Anti-neutrophil cytoplasmic antibodies (ANCAs), ds-DNA-antibody, $B 2$ 
glycoprotein-antibody, cardiolipin antibody IgG and IgM, and serology for Lues and Borrelia; assessment of thrombophilia-related markers including factor-V-Leiden mutation, methylenetetrahydrofolate-reductase mutation, prothrombin mutation and a test for Fabry disease. Moreover, depending on the previous findings, the patients might receive additional diagnostic procedures. In patients with PFO detection, we aim for a colour-coded duplex sonography of the lower extremity veins to rule out deep vein thrombosis (DVT). In case of clinical suspicion, a CT-angiography is performed to rule out pulmonary artery embolism (PE). The additional blood tests are performed in selected patients based on national and international guidelines and in house standard operating procedures.

\section{ICM implantation}

Implantation of an ICM is sought in all ESUS patients if 72 hours of automatic AF detection or Holter ECG recording did not return a diagnosis of AF. However, due to each individual patient's conditions (eg, reduced life expectancy due to patient's high age, severe stroke, an underlying malignant disease), the final decision for or against the implantation of an ICM is made by the treating physicians. The recording time takes place according to the lifetime of the ICM, which is expected to be 3-4years until the explantation.
Interdisciplinary diagnosis and treatment algorithm of ESUS patients

Patients $<60$ years of age (group 1) are assessed for the presence of a PFO. If a relevant PFO is absent, these patients are processed further along with patients $\geq 60$ years of age (group 2). In group 1 patients with a relevant $\mathrm{PFO}$ and an indication for lifelong $\mathrm{OAC}$ ( $\mathrm{eg}$, in the case of PE and/or DVT and relevant thrombophilia), the algorithm reaches a destination. In group 1 patients with a relevant $\mathrm{PFO}$ but without an indication for lifelong OAC, PFO closure is aimed for within the next 2-6 months in accordance with recent recommendations. ${ }^{8-10}$ These patients receive an ICM to rule out AF prior to PFO closure and are treated with ASA for secondary stroke prevention until PFO closure and thereafter. Group 1 patients with a relevant PFO are further stratified according to their individual risk for $\mathrm{AF}$ as described above. If the risk for $\mathrm{AF}$ is judged to be low and no $\mathrm{AF}$ is detected by ICM interrogation or clinically, the PFO will be closed within 2-6 months. If the risk for AF is judged to be high, we extend the observation period for ICM-based and clinical AF detection for AF up to 3 years before the PFO is closed.

For patients $\geq 60$ years (group 2 ) and those in group 1 without a relevant PFO, an ICM is implanted and ASA is prescribed for secondary stroke prevention. In case of AF

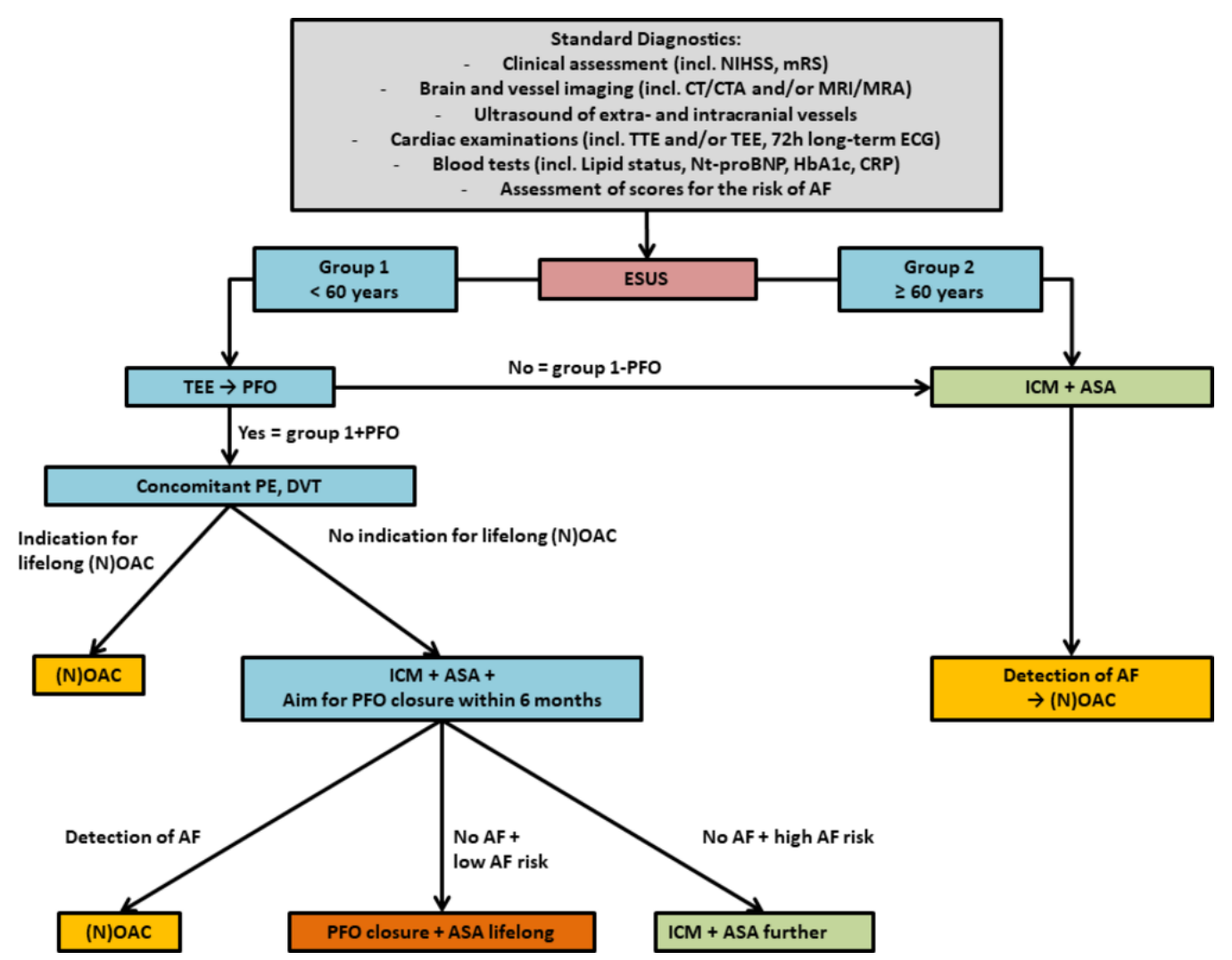

Figure 1 Diagnostic and therapeutic pathway. AF, atrial fibrillation; ASA, acetylsalicylic acid; CTA, CT-angiography; ICM, implantable cardiac monitor; MR-A, MRI-angiography; mRS, modified Rankin Scale; NIHSS, National Institutes of Health Stroke Scale; (N)OAC, (new) oral anticoagulation; PFO, patent foramen ovale; TEE, transesophageal echocardiography; TTE, transthoracic echocardiogram. 
detection, the secondary prevention regimen is changed to OAC (see figure 1).

\section{Follow-up}

All patients receive a standardised follow-up irrespective of their group allocation at 3,12 and 36 months after the index stroke. Follow-up is warranted by regular outpatient visits or via structured telephone interviews as defined in the study protocol.

\section{Clinical and neurological assessment}

The neurological clinical assessment evaluates the patients with respect to stroke recurrence, occurrence of other vascular diseases and the presence of cardiovascular risk factors. For the assessment of functional status, NIHSS and mRS are determined. The current medication is documented.

\section{Cardiological assessment}

The cardiological clinical assessment during follow-up aims to detect AF by means of ICM interrogation or Holter ECGs. In case of a relevant PFO, the decision for extended monitoring or closure is evaluated and documented. The $\mathrm{CHA}_{2} \mathrm{DS}_{2}$-VASc score is assessed at each visit.

\section{Initial baseline data}

Initial baseline and follow-up data of first 142 patients are presented in table 1 .

\section{DISCUSSION}

There is an urgent need to individualise and thereby improve the management of patients with stroke of undetermined cause and ESUS. Comprehensive guidelines for these entities are still lacking and recent randomised clinical trials did not fulfil the expectations placed in them. In addition to the recent ESUS trials, which did not support the prescription of OAC for secondary stroke prevention, at least two additional clinical trials testing apixaban compared with ASA are ongoing and their results are highly anticipated; yet, it will be several more years until results become available. ${ }^{21} 22$

A problem inherent to the definition of ESUS in general is the compression of several possibly different clinical entities into one diagnosis. It may thus be that this compression contributes to the inability of the recent ESUS trials to identify subgroups of patients actually benefiting from OAC for secondary stroke prevention. However, important clinical questions in some of the possible subgroups remain unresolved. Examples include ESUS patients with concomitant PFO, the relevance of prolonged $\mathrm{AF}$ detection, and the consideration of additional clinical risk stratification for AF. In the RESPECTESUS trial, a predefined subgroup analysis of patients $>75$ years of age suggested that dabigatran was superior to ASA in secondary stroke prevention. Whereas the relation cannot be verified for causality based on the available data, the results strengthen the relevance of and need for extended cardiac diagnostics and risk stratification to better understand the inherently heterogeneous ESUS
Table 1 Baseline characteristics of the first 142 patients in Catch-Up-ESUS

\begin{tabular}{|c|c|}
\hline \multicolumn{2}{|l|}{ Assessed characteristic } \\
\hline Patients (n) & 142 \\
\hline Sex-male (n, \%) & $92(65)$ \\
\hline Age-years, mean (SD) & $66,5 \pm 13$ \\
\hline Above 60 years, $\mathrm{n} 0$ & $96(68)$ \\
\hline \multicolumn{2}{|l|}{ Type of ischaemia } \\
\hline Stroke, n (\%) & $130(92)$ \\
\hline TIA, n (\%) & $12(8)$ \\
\hline pmRS; median (IQR) & $0(0,0)$ \\
\hline pmRS 0-2, n (\%) & $141(98)$ \\
\hline pmRS 3-5, n (\%) & $1(1)$ \\
\hline $\mathrm{mRS}$ at admission, median (IQR) & $2(1,3)$ \\
\hline mRS 0-2, n (\%) & $94(65)$ \\
\hline mRS 3-5, n (\%) & $48(33)$ \\
\hline NIHSS at admission, median (IQR) & $2(1,4)$ \\
\hline NIHSS 0-4, n (\%) & $107(74)$ \\
\hline NIHSS 5-14, n (\%) & $25(17)$ \\
\hline NIHSS $\geq 15, \mathrm{n}(\%)$ & $10(7)$ \\
\hline $\mathrm{CHA}_{2} \mathrm{DS}_{2}$-VASc Score, median (IQR) & $4(3,5)$ \\
\hline TTE, n (\%) & $76(54)$ \\
\hline TEE, n (\%) & $62(44)$ \\
\hline Relevant PFO, n (\%) & $29(20)$ \\
\hline Relevant PFO $<60$ years, $n$ ( $\%$ in $<60$ years) & $22(48)$ \\
\hline Implantation of ICM, n (\%) & $87(61)$ \\
\hline \multicolumn{2}{|l|}{ Secondary prophylaxis (at admission) } \\
\hline Antiplatelet, n (\%) & $48(34)$ \\
\hline (N)OAC, n (\%) & $2(1.4)$ \\
\hline None, n (\%) & $92(65)$ \\
\hline \multicolumn{2}{|l|}{ Secondary prophylaxis (at discharge) } \\
\hline Antiplatelet, n (\%) & $124(87)$ \\
\hline (N)OAC, n (\%) & $18(13)$ \\
\hline \multicolumn{2}{|l|}{ Cardiovascular risk factors } \\
\hline Arterial hypertension, $\mathrm{n}(\%)$ & $100(70)$ \\
\hline Diabetes mellitus, $\mathrm{n}(\%)$ & $25(18)$ \\
\hline Smoking, n (\%) & $48(34)$ \\
\hline $\begin{array}{l}\text { Positive family history of cardiovascular } \\
\text { conditions, } \mathrm{n}(\%)\end{array}$ & $14(10)$ \\
\hline Hypercholesterolemia, n (\%) & $41(29)$ \\
\hline
\end{tabular}

ASA, acetylsalicylic acid; CTA, CT-angiography; ICM, implantable cardiac monitor; MR-A, MRI-angiography; mRS, modified Rankin Scale; n, number; NIHSS, National Institutes of Health Stroke Scale;(N)OAC, (new) oral anticoagulation; PFO, patent foramen ovale; pmRS, premorbid modified Rankin Scale; TEE, transesophageal echocardiography; TIA, transient ischaemic attack; TTE, transthoracic echocardiogram.

patient population. In our registry, we aimed to overcome these issues, at least in part, by stratifying for relevant age groups and by extending the poststroke AF search duration. The expected results might help to better identify patients who would benefit from OAC. 
In response to the advantage of $\mathrm{PFO}$ closure in ESUS patients $<60$ years of age, a new management guideline on cryptogenic stroke and PFO was published by the German Society of Neurology. According to these guidelines, all 'patients between 16 and 60 years of age with a cryptogenic ischemic stroke and PFO with moderate or pronounced right-left shunt after neurological and cardiologic clarification are recommended a PFO occlusion' (https://www.dgn.org/leitlinien/3637-ll030-142-kryptogener-schlaganfall-und-offenes-foramenovale-2018). These national recommendations are also mirrored internationally. ${ }^{23}$ However, the guidelines do not comprehensively cover the extent of neurological and cardiological assessment required prior to PFO closure, especially regarding detailed diagnostic procedures to rule out competing etiologies like AF, which might be diagnosed by prolonged ECG screening. In our Catch-Up-ESUS registry, a standardised search for AF is implemented for up to 6 months using an ICM prior to an interventional PFO closure. As the closure of a PFO is invasive and may result in complications or insufficient success, such undesired sequelae may be avoided in patients in whom an indication for lifelong OAC, for example, due to $\mathrm{AF}$ can be ascertained. In addition, the group of patients $<60$ years of age, currently represented in clinical trials, is very heterogeneous. It ranges from some very young patients with $\mathrm{PFO}$ and without any cardiovascular risk factors to middle-aged patients with numerous cardiovascular risk factors. Increasing the likelihood of a cardioembolic cause of the stroke, as done by using the RoPE score applied in the context of Catch-Up-ESUS, may be helpful in identifying patients for whom PFO closure might be most beneficial. ${ }^{20}$ Also the attempt to rule out AF prior to a PFO closure and the possibility to extend the search for AF following PFO closure may be beneficial for patients

In summary, our open-label, investigator-initiated, prospective, monocentric, observational registry study will be helpful to answer urgent questions regarding the management of ESUS patients with respect to a stratified diagnosis and treatment.

However, our study has some limitations: first, most data for our registry are collected as part of the clinical routine. Therefore, we expect a certain loss of data in the course of data collection. In addition, we do not expect all patients to be treated according to our predefined algorithm because any clinical decision remains the sole responsibility of the treating physician. Our registry currently comprises single-centre data, limiting the expected number of enrolled individuals to currently 150-200 ESUS patients per year.

Together, our study addresses important and yet unanswered questions regarding ESUS patients. A more standardised and uniform diagnostic workup is urgently needed. The cooperation between neurologists and cardiologists represents a very promising approach in managing ESUS patients, especially in the context of prolonged rhythm monitoring and PFO detection and
PFO closure. We expect our study to contribute to a more individualised approach in ESUS patients in the future.

\section{Ethics and dissemination}

Catch-Up-ESUS is conducted in accordance with the Declaration of Helsinki. The study has been registered at www.clinicaltrialsregister.gov. All patients will have to give written informed consent or, if unable to give consent themselves, their legal guardian will have to provide written informed consent for their participation. The first observation period of the registry study is 1 year, followed by the first publication of the results including follow-up of the patients. Further publications will be considered according the predefined individual follow-up dates of the stroke patients up to 36 months.

\section{Author affiliations}

${ }^{1}$ Department of Neurology, Ludwig-Maximilians-Universitaet (LMU) Muenchen, Muenchen, Bayern, Germany

${ }^{2}$ German Center for Vertigo and Balance Disorders, Ludwig-Maximilians-Universitaet (LMU) Muenchen, Muenchen, Bayern, Germany

${ }^{3}$ Department of Medicine 1, Ludwig-Maximilians-Universitaet (LMU) Muenchen, Muenchen, Bayern, Germany

${ }^{4}$ German Centre for Cardiovascular Research (DZHK), partner site: Munich Heart Alliance, Muenchen, Bayern, Germany

${ }^{5}$ Institute for Stroke and Dementia Research (ISD), Ludwig-Maximilians-Universität (LMU) Muenchen, Muenchen, Bayern, Germany

Contributors All authors have read and approved the manuscript before submission. JH drafting/revising of the manuscript for content, including medical writing, study concept, design, interpretation of data, acquisition of data, development of clinical algorithm. KF revising of the manuscript for content, including medical writing, study concept, design, interpretation of data, acquisition of data. CK, KM, CL and RF revising manuscript for content, including medical writing. AST and FAW revising manuscript for content, including medical writing, acquisition of data. SK revising manuscript for content, including medical writing, acquisition of data, study concept, development of clinical algorithm. MFS revising manuscript for content, including medical writing, acquisition of data, study concept, development of clinical algorithm. LK drafting/revising of the manuscript for content, including medical writing, study concept, design, interpretation of data, acquisition of data, development of clinical algorithm.

Funding This research received no specific grant from any funding agency in the public, commercial or not-for-profit sectors.

Competing interests None declared.

Patient consent for publication Not required.

Ethics approval The study has been approved by the responsible ethics committee at the Ludwig Maximilian University (LMU), Munich, Germany (project number 17-685).

Provenance and peer review Not commissioned; externally peer reviewed.

Open access This is an open access article distributed in accordance with the Creative Commons Attribution Non Commercial (CC BY-NC 4.0) license, which permits others to distribute, remix, adapt, build upon this work non-commercially, and license their derivative works on different terms, provided the original work is properly cited, appropriate credit is given, any changes made indicated, and the use is non-commercial. See: http://creativecommons.org/licenses/by-nc/4.0/.

ORCID iD

Katharina Feil http://orcid.org/0000-0002-4566-712X

\section{REFERENCES}

1 Adams HP, Bendixen BH, Kappelle LJ, et al. Classification of subtype of acute ischemic stroke. definitions for use in a multicenter clinical trial. TOAST. trial of ORG 10172 in acute stroke treatment. Stroke 1993;24:35-41. 
2 Hart RG, Diener H-C, Coutts SB, et al. Embolic strokes of undetermined source: the case for a new clinical construct. Lancet Neurol 2014;13:429-38.

3 Hart RG, Sharma M, Mundl H, et al. Rivaroxaban for stroke prevention after embolic stroke of undetermined source. $N$ Engl $J$ Med 2018;378:2191-201.

4 World stroke Congress Abstracts, 2018. Int J Stroke 2018;13:3-217.

5 Homma S, Sacco RL. Patent foramen ovale and stroke. Circulation 2005;112:1063-72.

6 Alsheikh-Ali AA, Thaler DE, Kent DM. Patent foramen ovale in cryptogenic stroke: incidental or pathogenic? Stroke 2009;40:2349-55.

7 Katsanos AH, Psaltopoulou T, Sergentanis TN, et al. Transcranial Doppler versus transthoracic echocardiography for the detection of patent foramen ovale in patients with cryptogenic cerebral ischemia: a systematic review and diagnostic test accuracy meta-analysis. Ann Neurol 2016;79:625-35.

8 Mas J-L, Derumeaux G, Guillon B, et al. Patent foramen ovale closure or anticoagulation vs. antiplatelets after stroke. N Engl J Med 2017;377:1011-21.

9 Søndergaard L, Kasner SE, Rhodes JF, et al. Patent foramen ovale closure or antiplatelet therapy for cryptogenic stroke. N Engl J Med 2017:377:1033-42.

10 Saver JL, Carroll JD, Thaler DE, et al. Long-Term outcomes of patent foramen ovale closure or medical therapy after stroke. N Engl J Med 2017:377:1022-32.

11 Sanna T, Diener H-C, Passman RS, et al. Cryptogenic stroke and underlying atrial fibrillation. N Engl J Med 2014;370:2478-86.

12 Higgins P, MacFarlane PW, Dawson J, et al. Noninvasive cardiac event monitoring to detect atrial fibrillation after ischemic stroke: a randomized, controlled trial. Stroke 2013:44:2525-31.

13 Gladstone DJ, Spring M, Dorian P, et al. Atrial fibrillation in patients with cryptogenic stroke. N Engl J Med 2014;370:2467-77.

14 Wachter R, Gröschel K, Gelbrich G, et al. Holter-electrocardiogrammonitoring in patients with acute ischaemic stroke (FindAFRANDOMISED): an open-label randomised controlled trial. Lancet Neurol 2017;16:282-90.
15 Poli S, Diedler J, Härtig F, et al. Insertable cardiac monitors after cryptogenic stroke--a risk factor based approach to enhance the detection rate for paroxysmal atrial fibrillation. Eur $J$ Neurol 2016;23:375-81.

16 Piepoli MF, Hoes AW, Agewall S, et al. 2016 European Guidelines on cardiovascular disease prevention in clinical practice: The Sixth Joint Task Force of the European Society of Cardiology and Other Societies on Cardiovascular Disease Prevention in Clinical Practice (constituted by representatives of 10 societies and by invited experts) Developed with the special contribution of the European Association for Cardiovascular Prevention \& Rehabilitation (EACPR). Atherosclerosis 2016;252:207-74.

17 Gage BF, van Walraven C, Pearce L, et al. Selecting patients with atrial fibrillation for anticoagulation. Circulation 2004;110:2287-92.

18 Luchner A, Birner C, Laufs U. Zwei kardiale Marker werden "erwachsen": Die B-Typ-natriuretischen Peptide haben sich in der Notfalldiagnostik der Herz insuffizienz etabliert. Sie sind außerdem starke Risikoprädiktoren. Deutsches Ärzteblatt 2016;113.

19 Laufs U, Anker SD, Falk V, et al. Kommentar zu den Leitlinien Der Europäischen Gesellschaft für Kardiologie (ESC) Zur Diagnostik und Behandlung Der akuten und chronischen Herzinsuffizienz. Kardiologe 2017;11:183-92.

20 Kent DM, Ruthazer R, Weimar C, et al. An index to identify strokerelated vs incidental patent foramen ovale in cryptogenic stroke. Neurology 2013;81:619-25.

21 Geisler T, Poli S, Meisner C, et al. Apixaban for treatment of embolic stroke of undetermined source (ATTICUS randomized trial): rationale and study design. Int J Stroke 2017;12:985-90.

22 Kamel H, Longstreth WT, Tirschwell DL, et al. The atrial cardiopathy and antithrombotic drugs in prevention after cryptogenic stroke randomized trial: rationale and methods. Int J Stroke 2019;14.

23 Kuijpers T, Spencer FA, Siemieniuk RAC, et al. Patent foramen ovale closure, antiplatelet therapy or anticoagulation therapy alone for management of cryptogenic stroke? a clinical practice guideline. BMJ 2018;362. 